CLINICAL STUDY

\title{
Effect of a 4 week physical training program on plasma concentrations of inflammatory markers in patients with abnormal glucose tolerance
}

\author{
Andreas Oberbach ${ }^{1,2}$, Anke Tönjes ${ }^{2}$, Nora Klöting ${ }^{2}$, Mathias Fasshauer ${ }^{2}$, Jürgen Kratzsch ${ }^{3}$, Martin W Busse ${ }^{4}$, \\ Ralf Paschke ${ }^{2}$, Michael Stumvoll ${ }^{2}$ and Matthias Blüher ${ }^{1,2}$ \\ ${ }^{1}$ Junior Research Group NO3, IZKF Leipzig, Faculty of Medicine, ${ }^{2}$ Department of Internal Medicine III, ${ }^{3}$ Institute of Clinical Chemistry and \\ Pathobiochemistry and ${ }^{4}$ Institute of Exercise Medicine, University of Leipzig, Ph.-Rosenthal-Str. 27, D-04103 Leipzig, Germany \\ (Correspondence should be addressed to M Blüher; Email: bluma@medizin.uni-leipzig.de)
}

\begin{abstract}
Objective: Subclinical chronic inflammation could be a unifying factor linking type 2 diabetes (T2D) and atherosclerosis. The beneficial effects of physical activity on a reduced risk of coronary heart disease could at least in part be mediated by improved markers of inflammation.

Research design and methods: The aim of this study was to determine the effect of 4 weeks of physical training on plasma concentrations of interleukin (IL)-6, C-reactive protein (CRP), adiponectin and IL-10 in 60 individuals with normal glucose tolerance, impaired glucose tolerance (IGT) or T2D. Results: In patients with IGT and T2D, significant improvement in body fat, fitness level, glucose metabolism and insulin sensitivity after 4 weeks of physical training was associated with significantly improved plasma concentrations of adiponectin and CRP, but not IL-6. Regression analysis demonstrated only for the anti-inflammatory parameters adiponectin and IL-10 a significant relationship with the decrease in fasting plasma glucose, whereas changes in IL-6 and CRP were not significantly related to changes in fasting plasma glucose, body fat, maximal oxygen uptake, or insulin sensitivity. In a multivariate linear regression analysis, only changes in circulating adiponectin, fasting plasma glucose and percentage body fat were determinants of changes in insulin sensitivity.

Conclusions: Physical training was associated with a near normalization of adiponectin and CRP plasma concentrations in subjects with IGT and T2D. Increased insulin sensitivity after training was most strongly related to changes in adiponectin plasma concentrations, in fasting plasma glucose and percentage body fat, whereas changes in IL-6, IL-10 and CRP plasma concentrations did not significantly contribute to improved insulin sensitivity.
\end{abstract}

European Journal of Endocrinology 154 577-585

\section{Introduction}

Patients with type 2 diabetes mellitus (T2D) have an increased risk for premature atherosclerosis $(1,2)$. Chronic systemic inflammation is recognized as a part of both atherosclerosis (3) and T2D (4) and could therefore represent the common pathogenic factor in the development of these diseases. Altered plasma concentrations of inflammatory mediators may be pathogenic by inducing systemic endothelial dysfunction. Several studies have demonstrated that elevated markers of inflammation such as interleukin (IL)-6 $(5,6)$ and C-reactive protein (CRP) $(6-8)$, as well as decreased anti-inflammatory factors including adiponectin $(9-11)$ and IL-10 $(12-14)$ are predictors of insulin resistance and T2D. However, there are other studies that could not show changes in IL-6, CRP and adiponectin plasma concentrations after regular physical exercise interventions (15-17). Physical activity is associated with reduced risk of cardiovascular disease (CVD), cardiovascular death and total mortality in men with T2D (18). The beneficial effects of physical activity on a reduced CVD risk could at least in part be mediated by improved markers of inflammation. It has been suggested that exercise produces a shortterm inflammatory response, whereas both cross-sectional comparisons and longitudinal exercise training studies demonstrate a long-term anti-inflammatory effect (19). In nondiabetic, insulin-resistant individuals, it was recently shown that exercise training is not associated with improved CRP or adiponectin levels (20). However, the effects of exercise on inflammatory 
parameters in patients with T2D need to be further investigated. It is still unclear whether long-term exercise-associated anti-inflammatory effects are entirely explained by changes in body fat content, insulin sensitivity, fitness level or glucose metabolism in individuals with impaired glucose metabolism. Moreover, exerciseinduced changes in inflammatory markers could contribute to the improvement of insulin sensitivity after physical training.

We therefore investigated the effects of a 4 week physical training program on plasma concentrations of IL-6, adiponectin, CRP and IL-10. We further asked whether changes in these parameters go beyond that expected from changes in obesity. We finally analyzed the relationship of changes of these inflammation and endothelial dysfunction markers with insulin sensitivity.

\section{Research design and methods}

\section{Subjects}

Sixty Caucasian men and women (mean age 45.2 \pm 3.9 years) were enrolled in a supervised physical training program. Each subject underwent three times a week a 60 min training program, which was monitored and documented by two trainers. Each training session consisted of $20 \mathrm{~min}$ warming and cool-down periods, $20 \mathrm{~min}$ of running or biking, and $20 \mathrm{~min}$ of powertraining three times a week. In addition, participants performed $60 \mathrm{~min}$ of swimming on a separate day. The compliance rate was $100 \%$. These subjects had been randomly selected from more than 500 volunteers at the beginning of a training program, who were screened for impaired glucose tolerance (IGT) by a $75 \mathrm{~g}$ oral glucose-tolerance test (OGTT) and subsequently divided into three different groups according to American Diabetes Association (ADA) criteria (21), i.e. subjects with (i) normal glucose tolerance (NGT), (ii) IGT or (iii) T2D. Subjects with NGT were defined by a fasting plasma glucose $<6.0 \mathrm{mmol} / \mathrm{l}$ and a $120 \mathrm{~min}$ plasma glucose $<7.8 \mathrm{mmol} / \mathrm{l}$ after a $75 \mathrm{~g}$ oral glucose load (21). These subjects exhibited no family history of diabetes or T2D during pregnancy ( $n=20 ; 9$ males, 11 females). Subjects with IGT were defined by a fasting plasma glucose $<6.0 \mathrm{mmol} / \mathrm{l}$ and a $120 \mathrm{~min}$ plasma glucose $>7.8 \mathrm{mmol} / \mathrm{l}$ and $<11.1 \mathrm{mmol} / \mathrm{l}$ after a $75 \mathrm{~g}$ oral glucose load (21) ( $n=20 ; 9$ males, 11 females). Subjects with T2D were defined by a fasting plasma glucose $>7 \mathrm{mmol} / \mathrm{l}$ and/or a $120 \mathrm{~min}$ OGTT glucose $>11.1 \mathrm{mmol} / \mathrm{l}$ (21) ( $n=20 ; 11$ males, 9 females). All subjects fulfilled the following inclusion criteria: (i) absence of any acute or chronic inflammatory disease; (ii) undetected glutamic acid decarboxylase; (iii) no medical history of hypertension, i.e. their systolic blood pressure was $<140 \mathrm{mmHg}$ and diastolic blood pressure was $<90$ mmHg; (iv) no clinical evidence of either CVD or peripheral artery disease; (v) no thyroid dysfunction; (vi) no concomitant medication intake, except for metformin ( $500 \mathrm{mg}$ twice daily) in the T2D group; (vii) no alcohol or drug abuse; and (viii) no pregnancy. In addition, there was no evidence of diabetic retinopathy or nephropathy in these patients. During the 4 week training period, constant daily caloric intake was reported by each subject using dietary diaries. The study was approved by the ethics committee of the University of Leipzig. All subjects gave written informed consent before taking part in the study.

\section{Measures of body fat content and OGTT}

At baseline and after 4 weeks of training, blood samples were taken for the measurement of IL-6, CRP, IL-10, adiponectin and standard laboratory parameters using the previously described methods (6). In addition dualenergy X-ray absorptiometry (DEXA) analyses and measurements of anthropometric parameters were performed. Body mass index (BMI) was calculated as weight $(\mathrm{kg})$ divided by squared height $(\mathrm{m})$. Waist and hip circumferences were measured and a waist-to-hip ratio (WHR) was calculated. Percentage body fat was measured by DEXA. The OGTT was performed according to ADA criteria (21). Three days prior to the OGTT the patients documented a high carbohydrate diet. The OGTT was performed after an overnight fast with a $75 \mathrm{~g}$ standardized glucose solution (Glucodex Solution $75 \mathrm{~g}$; Merieux, Montreal, Canada). Venous blood samples were taken at 0,60 and 120 min for measurements of plasma glucose concentrations. Insulin sensitivity was assessed with the euglycemic-hyperinsulinemic clamp method as previously described $(22,23)$.

\section{Maximal exercise test}

All subjects completed a graded bicycle-ergometer test to volitional exhaustion by measuring maximal oxygen uptake $\left(\mathrm{VO}_{2} \max \right)$ with an automated open circuit gas analysis system at baseline. The highest oxygen uptake/minute reached was defined as the $\mathrm{VO}_{2}$ max. Individuals in the supervised physical training subgroup $(n=60)$ completed an additional maximal exercise test after 4 weeks of physical training.

\section{Assays}

Basal, fasting blood samples were taken after an overnight fast to determine glucose, insulin, and standard laboratory parameters. Plasma concentrations of insulin, C-peptide, free fatty acid (FFA) and leptin were measured as previously described (22). Plasma adiponectin was assessed using a commercially available RIA kit (Linco Research, St Charles, MO, USA). Serum samples for CRP, IL- 6 and IL-10 were stored at $-80^{\circ} \mathrm{C}$ and assayed in triplicate. High-sensitivity ELISAs were used for IL-6 and IL-10 (Quantikine IL-6, IL-10; R\&D Systems, Oxford, UK) as previously described (6), and 
the high-sensitivity CRP assay was by immunonephelometry (Dade-Behring, Milan, Italy). Both interassay and intraassay coefficients of variation were $<5 \%$.

\section{Statistical analysis}

Data are shown as means \pm S.D. unless stated otherwise. Prior to statistical analysis, non-normally distributed parameters were log transformed to approximate a normal distribution. The following statistical tests were used: paired Student's t-test, Chi quadrate test, and Pearson's simple correlation. Linear relationships were assessed by least square regression analysis. Multivariate linear relationships were assessed by a general linear model. Statistically analysis was performed using SPSS version 12.0 (SPSS, Chicago, IL, USA). In order to determine whether changes in inflammatory parameters are proportional or disproportional to training-induced changes in total body fat, increased fitness level and insulin sensitivity, random intercept models have been calculated, which considered changes of the inflammatory markers and their interaction with changes in $\mathrm{VO}_{2}$ max, glucose infusion rate during the steady-state of the clamp and percent body fat. These calculations were performed using the software 'Proc mixed' of the SAS 9.1 package (SAS Institute, Inc., Cary, NC, USA).

\section{Results}

\section{Subjects}

The 60 Caucasian men and women were studied either together or after being divided into three different groups, i.e. subjects with NGT $(n=20)$, or IGT $(n=20)$ or T2D $(n=20)$ (Table 1$)$. Subjects reported daily caloric intake and macronutrient composition was constant during the study period (Table 1). The training effect was confirmed by a significant improvement in $\mathrm{VO}_{2}$ max in all groups (Table 1). Four weeks of physical training resulted in significant decreases in BMI, WHR and percent body fat in all glucose-tolerance groups (Table 1). Moreover, in the IGT and T2D groups, whole-body glucose uptake during the steady-state of a euglycemic-hyperinsulinemic clamp significantly improved after 4 weeks of physical training (Table 1). In patients with $\mathrm{T} 2 \mathrm{D}$, there was an additional significant decrease in fasting plasma glucose concentrations after 4 weeks of physical training, whereas no such difference was detected in the NGT and IGT groups (Table 1).

\section{Correlation of chronic inflammatory markers with parameters of obesity, glucose metabolism, insulin sensitivity and fitness level}

Plasma IL-6 concentration was significantly positively correlated with percent body fat (Fig. 1), BMI $\left(r^{2}=0.11 ; \quad P<0.001\right), \quad$ fasting plasma glucose $\left(r^{2}=0.1 ; \quad P<0.001\right)$ and insulin concentration $\left(r^{2}=0.2 ; \quad P<0.001\right)$. There was a significant negative correlation between IL- 6 plasma levels and $\mathrm{VO}_{2}$ max and glucose infusion rate during the steady-state of the euglycemic-hyperinsulinemic clamp (Fig. 1). There was a significant negative correlation between adiponectin plasma concentrations and percent body fat (Fig. 2), BMI $\left(r^{2}=0.1\right.$; $P<0.001)$, fasting plasma glucose $\left(r^{2}=0.1\right.$; $P<0.001)$ and insulin concentrations $\left(r^{2}=0.2\right.$; $P<0.001)$. Adiponectin levels were significantly positively correlated with $\mathrm{VO}_{2}$ max and insulin sensitivity (Fig. 2). IL-10 plasma concentrations negatively correlated with percent body fat (Fig. 3), BMI $\left(r^{2}=0.05 ; \quad P<0.001\right), \quad$ fasting plasma glucose $\left(r^{2}=0.03 ; P<0.001\right)$ and insulin concentration $\left(r^{2}=0.07 ; P<0.001\right)$, whereas a significantly positive correlation was found between IL-10 levels and $\mathrm{VO}_{2} \mathrm{max}$ and glucose infusion rate during the steady-state of the euglycemic-hyperinsulinemic clamp (Fig. 3). CRP plasma concentration was significantly positively correlated with percent body fat (Fig. 4), BMI $\left(r^{2}=0.22 ; \quad P<0.001\right), \quad$ fasting plasma glucose $\left(r^{2}=0.14 ; P<0.001\right)$ and insulin concentration $\left(r^{2}=0.2 ; P<0.001\right)$. There was a significantly negative correlation between CRP plasma levels and $\mathrm{VO}_{2} \max$ and glucose infusion rate during the steady-state of the euglycemic-hyperinsulinemic clamp (Fig. 4).

\section{Effect of 4 weeks of physical training on markers of chronic inflammation}

Plasma adiponectin concentration was significantly increased after 4 weeks of physical training in the IGT and T2D groups (Fig. 5). Interestingly, 4 weeks of exercise did not have any effect on IL-6 plasma concentrations (Fig. 5). Moreover, plasma IL-10 concentrations were not significantly different between baseline and after 4 weeks of training. However, there was a tendency for increased IL-10 plasma concentrations in the IGT and T2D groups (Fig. 5). Plasma concentrations of CRP significantly decreased in all three glucose-tolerance groups (Fig. 5).

\section{Effect of training on changes in chronic inflammatory markers beyond that expected from changes in obesity, glucose metabolism, insulin sensitivity and fitness level}

Despite the significant decrease in percent body fat and increases in $\mathrm{VO}_{2} \max$ and insulin sensitivity, IL-6 plasma concentrations were unchanged after 4 weeks of physical training (Fig. 1). In contrast, the significant increase in adiponectin levels after 4 weeks of physical training (Fig. 5) is closely associated with improvement of insulin sensitivity and disproportionally exceeds the 
Table 1 Anthropometric and biochemical parameters (means \pm S.D.) at baseline and after 4 weeks of intensive physical training in subjects with NGT, IGT or T2D. Dietary record data are shown as means during the study for men $(\mathrm{M})$ and women $(\mathrm{F})$.

\begin{tabular}{|c|c|c|c|c|c|c|}
\hline & \multicolumn{2}{|c|}{ NGT $(n=20)$} & \multicolumn{2}{|c|}{ IGT $(n=20)$} & \multicolumn{2}{|c|}{ T2D $(n=20)$} \\
\hline & Baseline & Post-interventional & Baseline & Post-interventional & Baseline & Post-interventional \\
\hline BMI $\left(\mathrm{kg} / \mathrm{m}^{2}\right)$ & $24.2 \pm 3.1$ & $23.9 \pm 2.7^{*}$ & $29.8 \pm 3.9$ & $29.0 \pm 3.7^{*}$ & $31.3 \pm 3.1$ & $30.5 \pm 2.7^{*}$ \\
\hline Body weight (kg) & $69.6 \pm 14.0$ & $68.2 \pm 7.7^{*}$ & $87.6 \pm 16.4$ & $84.4 \pm 6.1^{*}$ & $94.6 \pm 19.6$ & $93.0 \pm 8.1^{*}$ \\
\hline WHR & $0.84 \pm 0.09$ & $0.81 \pm 0.08^{*}$ & $1.20 \pm 0.16$ & $1.17 \pm 0.15^{*}$ & $1.28 \pm 0.12$ & $1.24 \pm 0.11 *$ \\
\hline Fat mass (\%) & $24.5 \pm 3.1$ & $23.2 \pm 2.7^{*}$ & $34.9 \pm 8.2$ & $31.5 \pm 7.4^{*}$ & $38.1 \pm 7.9$ & $35.2 \pm 7.6^{\star}$ \\
\hline $\mathrm{FPG}(\mathrm{mmol} / \mathrm{l})$ & $5.1 \pm 0.5$ & $5.0 \pm 0.3$ & $5.6 \pm 0.5$ & $5.4 \pm 0.6$ & $6.2 \pm 0.6$ & $5.8 \pm 0.4^{*}$ \\
\hline $2 \mathrm{~h}$ OGTT glucose $(\mathrm{mmol} / \mathrm{l})$ & $5.9 \pm 0.76$ & $5.5 \pm 0.5$ & $9.4 \pm 0.8$ & $8.1 \pm 1.3^{*}$ & $13.0 \pm 1.5$ & $12.6 \pm 2.4$ \\
\hline FPI (pmol/l) & $66 \pm 34$ & $57 \pm 27$ & $695 \pm 493$ & $379 \pm 323^{*}$ & $319 \pm 212$ & $234 \pm 119$ \\
\hline Glucose infusion rate $(\mu \mathrm{mol} / \mathrm{kg} / \mathrm{min})$ & $76 \pm 17$ & $85 \pm 15$ & $19 \pm 9$ & $36 \pm 16^{*}$ & $21 \pm 9$ & $32 \pm 11 *$ \\
\hline $\mathrm{VO}_{2} \max (\mathrm{ml} / \mathrm{kg} / \mathrm{min})$ & $34.2 \pm 2.6$ & $36.1 \pm 3.2^{*}$ & $26.4 \pm 1.9$ & $28.1 \pm 1.4^{*}$ & $26.1 \pm 1.8$ & $27.5 \pm 1.9^{*}$ \\
\hline Triglyceride (mmol/l) & $1.4 \pm 0.4$ & $1.5 \pm 0.6$ & $2.5 \pm 0.1$ & $2.2 \pm 0.4^{*}$ & $3.0 \pm 0.1$ & $2.1 \pm 0.5^{\star}$ \\
\hline $\mathrm{FFA}(\mathrm{mmol} / \mathrm{l})$ & $0.41 \pm 0.19$ & $0.39 \pm 0.18$ & $0.53 \pm 0.24$ & $0.50 \pm 0.24$ & $0.56 \pm 0.26$ & $0.47 \pm 0.22$ \\
\hline \multicolumn{7}{|l|}{ Leptin (pmol/l) } \\
\hline M & $2.7 \pm 2.0$ & $2.6 \pm 1.7$ & $20.6 \pm 9.0$ & $21.1 \pm 7.5$ & $3.3 \pm 1.8$ & $3.5 \pm 2.0$ \\
\hline $\mathrm{F}$ & $6.0 \pm 2.7$ & $5.8 \pm 2.7$ & $42.2 \pm 23.8$ & $36.7 \pm 19.5$ & $9.0 \pm 1.7$ & $9.0 \pm 2.4$ \\
\hline Total cholesterol $(\mathrm{mmol} / \mathrm{l})$ & $4.6 \pm 0.4$ & $4.7 \pm 0.4$ & $5.3 \pm 0.5$ & $5.5 \pm 0.5$ & $5.6 \pm 0.7$ & $5.4 \pm 0.4$ \\
\hline Total HDL (mmol/l) & $1.6 \pm 0.4$ & $1.5 \pm 0.2$ & $1.2 \pm 0.3$ & $1.9 \pm 0.3^{*}$ & $1.2 \pm 0.3$ & $1.7 \pm 0.4^{*}$ \\
\hline Total LDL (mmol/l) & $2.4 \pm 0.4$ & $2.0 \pm 0.4^{*}$ & $3.3 \pm 0.4$ & $2.7 \pm 0.6^{*}$ & $3.4 \pm 0.8$ & $2.8 \pm 0.7^{*}$ \\
\hline \multicolumn{7}{|l|}{ Dietary record data $\mathrm{M} / \mathrm{F}$} \\
\hline Energy intake (kcal/day) & \multirow{2}{*}{\multicolumn{2}{|c|}{$3538 \pm 802 / 2537 \pm 512$}} & \multicolumn{2}{|c|}{$3633 \pm 538 / 2987 \pm 674$} & \multicolumn{2}{|c|}{$3942 \pm 967 / 2818 \pm 635$} \\
\hline Carbohydrate (\% of energy) & & & \multicolumn{2}{|c|}{$38.8 \pm 7.3 / 46.4 \pm 6.4$} & \multicolumn{2}{|c|}{$41.8 \pm 7.7 / 43.4 \pm 9.1$} \\
\hline Fat (\% of energy) & \multirow{2}{*}{\multicolumn{2}{|c|}{$35.4 \pm 6.7 / 31.3 \pm 8.5$}} & \multicolumn{2}{|c|}{$43.6 \pm 9.1 / 35.4 \pm 9.3$} & \multicolumn{2}{|c|}{$39.8 \pm 4.3 / 39.4 \pm 6.4$} \\
\hline Protein (\% of energy) & $19.5 \pm 5.2 / 19.0 \pm 4.5$ & & \multicolumn{2}{|c|}{$17.6 \pm 5.8 / 18.2 \pm 3.9$} & \multicolumn{2}{|c|}{$18.4 \pm 5.9 / 17.2 \pm 3.1$} \\
\hline
\end{tabular}

${ }^{*} P<0.05$ for baseline vs after 4 weeks of intensive physical training within each group.

HDL, high-density lipoprotein; LDL, low-density lipoprotein; FPG, fasting plasma glucose; FPI, fasting plasma insulin. 
beneficial effects of reduced percent body fat and increased fitness level $\left(\mathrm{VO}_{2} \max \right)$ (Fig. 2). Increased IL-10 plasma concentration (Fig. 5) were proportional to the improvement of body fat content, $\mathrm{VO}_{2}$ max and insulin sensitivity after 4 weeks of training (Fig. 3).
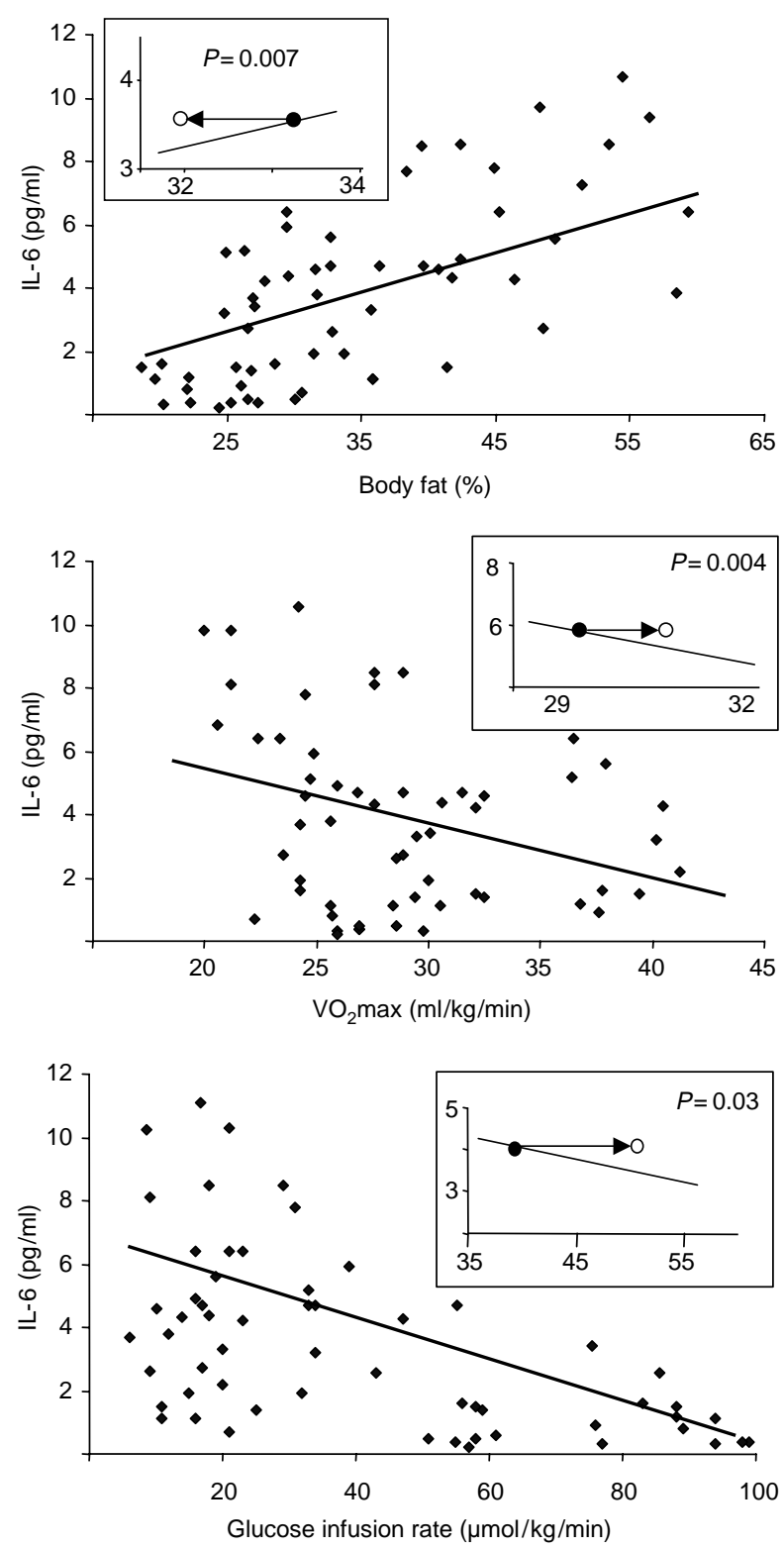

Figure 1 Correlation of plasma IL-6 concentrations with (upper panel) total body fat, (middle panel) maximal aerobic capacity $\left(\mathrm{VO}_{2} \mathrm{max}\right)$, and (lower panel) glucose infusion rate during the steady-state of a euglycemic-hyperinsulinemic clamp. The inserts in each panel show changes of mean IL- 6 from baseline to after physical exercise concentrations. Closed circles represent baseline levels, open circles represent IL-6 concentrations after 4 weeks of intensive physical training $(n=60)$. $P$-values ( $t$-test) indicate whether IL-6 after-training concentrations are significantly different from the calculated after-training concentration derived from the regression curve equation. The direction of the changes is indicated by arrows.
The significant decrease in CRP levels after 4 weeks of intensive training (Fig. 5) disproportionally exceeds the expected effects from the improvement of insulin sensitivity, reduced percent body fat and increased fitness level $\left(\mathrm{VO}_{2} \max \right)$ (Fig. 4).
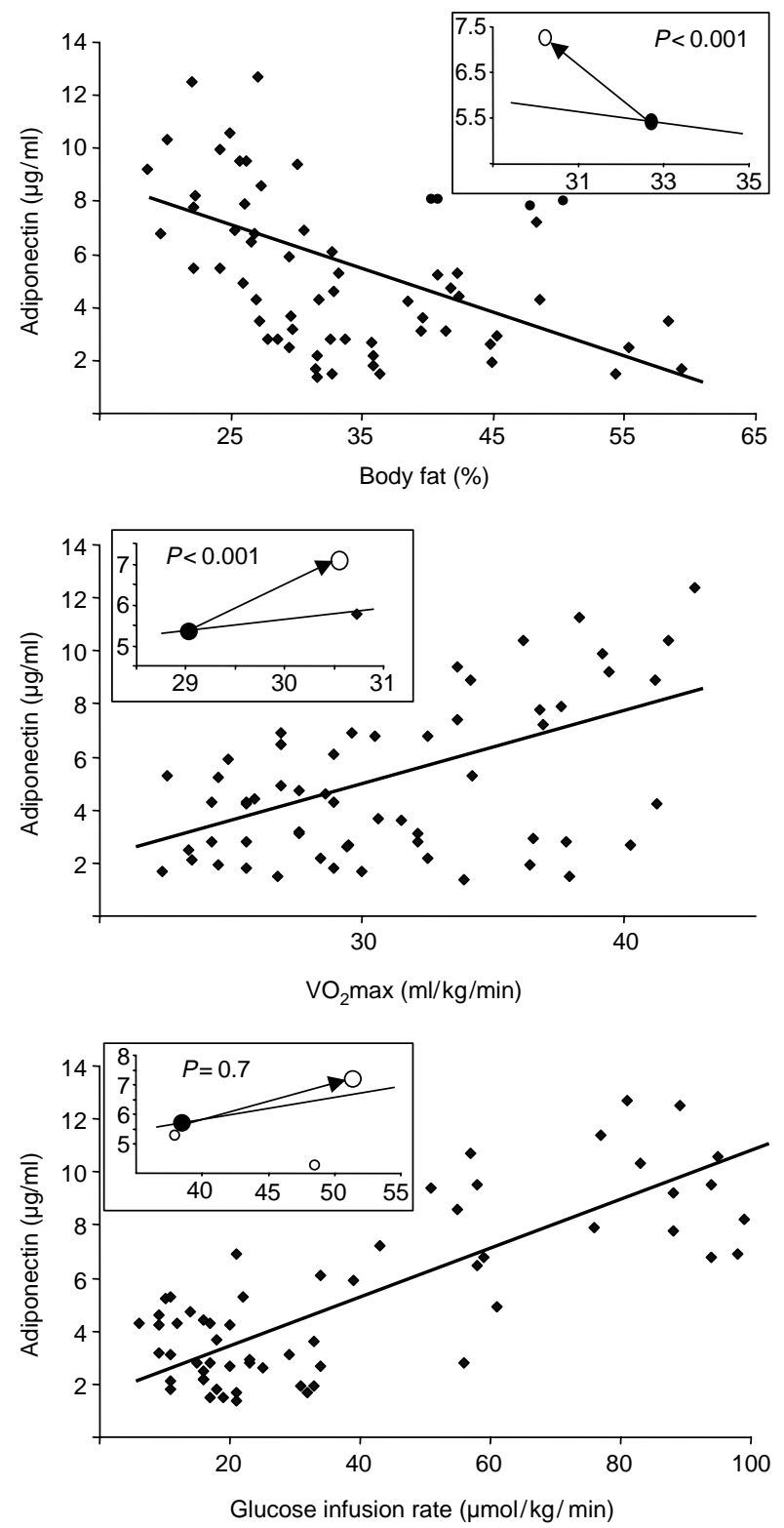

Figure 2 Correlation of plasma adiponectin concentrations with (upper panel) total body fat, (middle panel) maximal aerobic capacity $\left(\mathrm{VO}_{2} \mathrm{max}\right)$, and (lower panel) glucose infusion rate during the steady-state of a euglycemic-hyperinsulinemic clamp. The three inserts show changes of mean adiponectin from baseline to after physical exercise concentrations. Closed circles represent baseline levels, open circles represent adiponectin concentrations after 4 weeks of intensive physical training $(n=60)$. $P$-values $(t$-test) indicate whether adiponectin after-training concentrations are significantly different from the calculated after-training concentration derived from the regression curve equation. The direction of the changes is indicated by arrows. 


\section{Multivariate regression analyses}

The correlations found by simple linear regression analysis were further analyzed in more detail. Multivariate linear regression analysis of relative changes in percent body fat, $\mathrm{VO}_{2} \mathrm{max}$, and fasting plasma glucose as
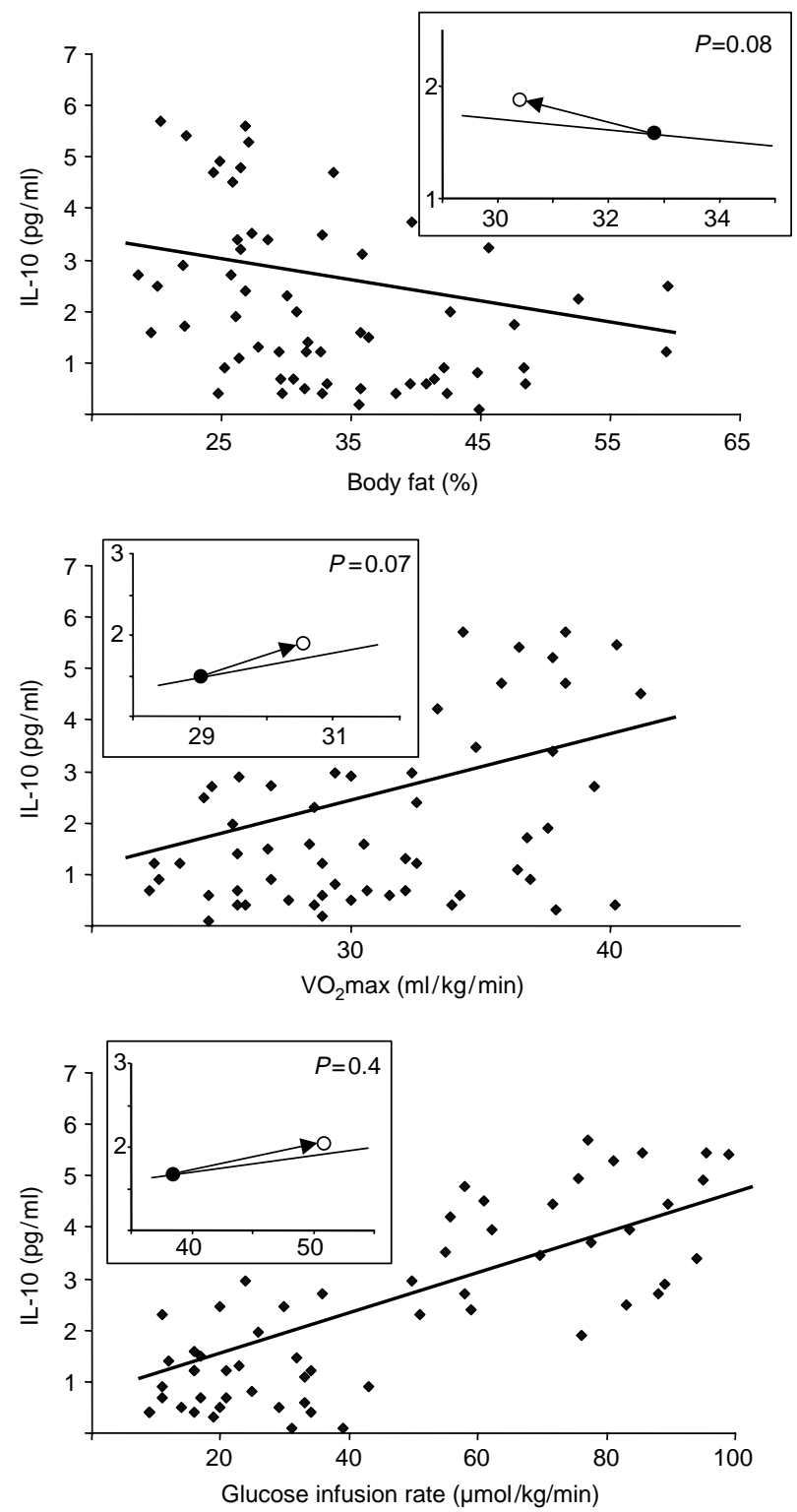

Figure 3 Correlation of plasma IL-10 concentrations with (upper panel) total body fat, (middle panel) maximal aerobic capacity $\left(\mathrm{VO}_{2} \mathrm{max}\right)$, and (lower panel) glucose infusion rate during the steady-state of a euglycemic-hyperinsulinemic clamp. The three inserts show changes of mean IL-10 from baseline to after physical exercise concentrations. Closed circles represent baseline levels, open circles represent IL-10 concentrations after 4 weeks of intensive physical training $(n=60)$. $P$-values ( $t$-test) indicate whether IL-10 after-training concentrations are significantly different from the calculated after-training concentration derived from the regression curve equation. The direction of the changes is indicated by arrows. predictors for the relative changes in adiponectin, CRP, IL-6 and IL-10 plasma concentrations revealed only changes in fasting plasma glucose as significant determinants of changes in adiponectin and IL-10 con-
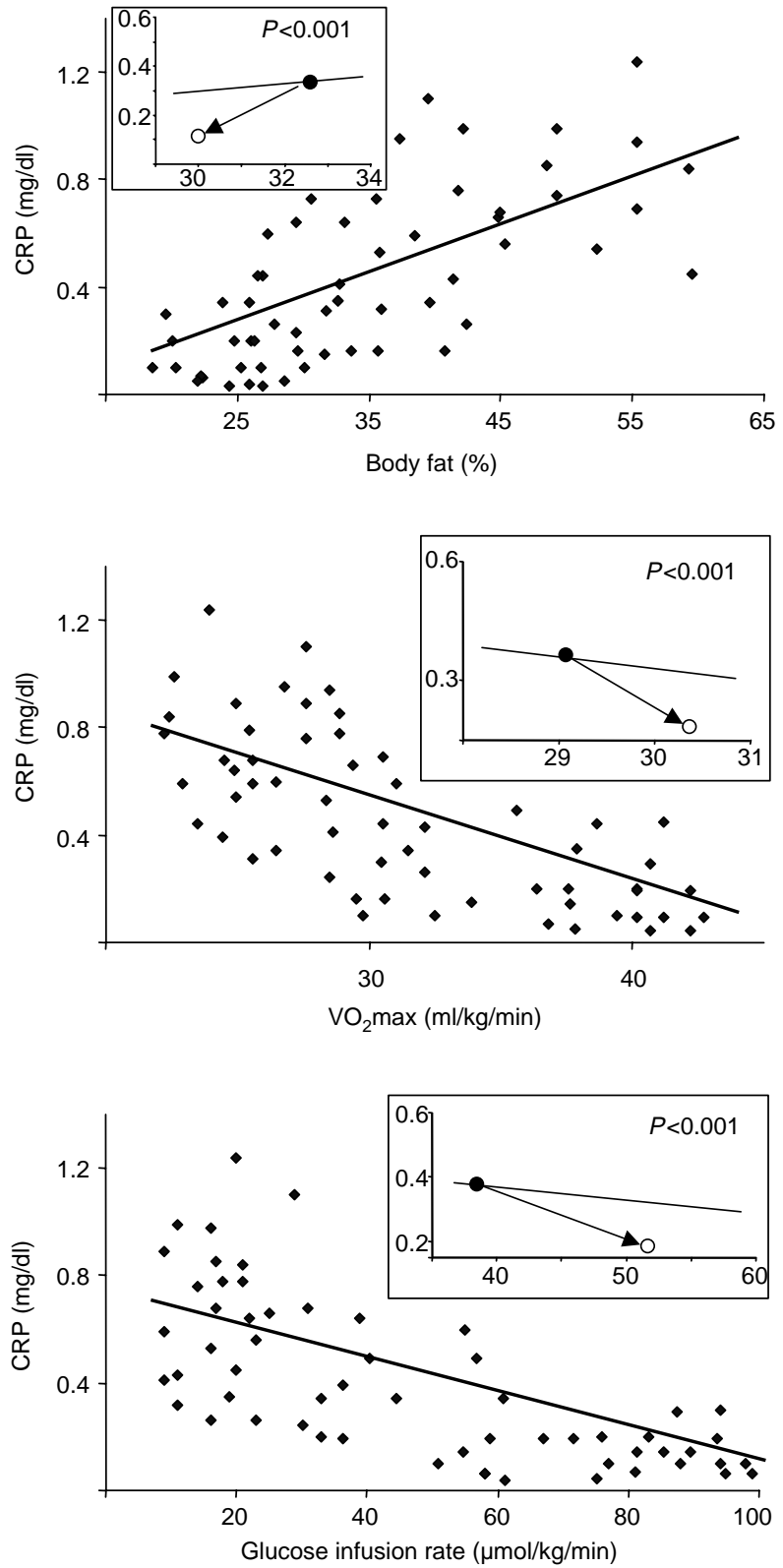

Figure 4 Correlation of plasma CRP concentrations with (upper panel) total body fat, (middle panel) maximal aerobic capacity $\left(\mathrm{VO}_{2} \mathrm{max}\right)$, and (lower panel) glucose infusion rate during the steady-state of a euglycemic-hyperinsulinemic clamp. The three inserts show changes of mean CRP from baseline to after physical exercise concentrations. Closed circles represent baseline levels, open circles represent CRP concentrations after 4 weeks of intensive physical training $(n=60)$. $P$-values $(t$-test) indicate whether CRP after-training concentrations are significantly different from the calculated after-training concentration derived from the regression curve equation. The direction of the changes is indicated by arrows. 
Adiponectin
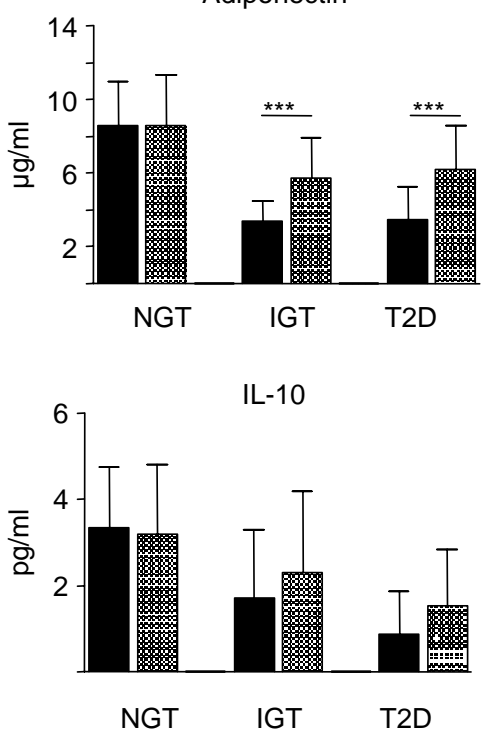

IL-6
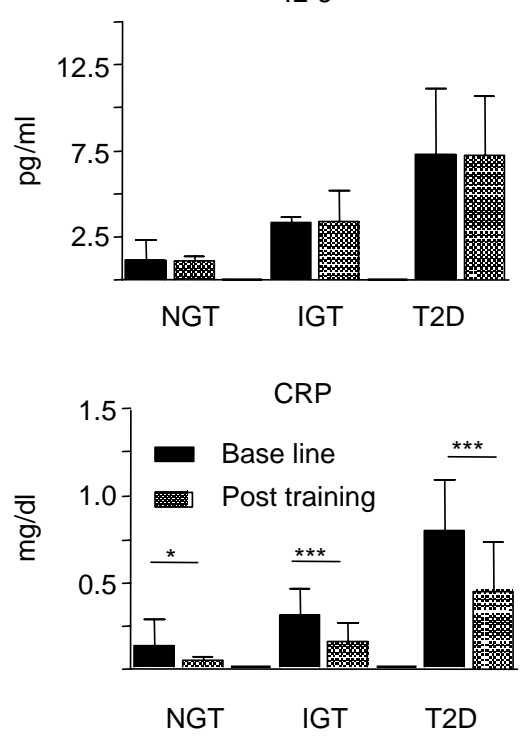

Figure 5 Baseline and post-interventional plasma concentrations of (mean \pm S.D.) adiponectin, IL-6, IL-10 and CRP in subjects with NGT $(n=20)$ and IGT $(n=20)$ and in patients with T2D $(n=20)$. * $P<0.05$, ${ }^{\star *} P<0.01,{ }^{* \star \star} P<0.001$ between baseline and after 4 weeks of intensive physical training. centrations after 4 weeks of physical training (Table 2). We further assessed the role of changes in percent body fat, $\mathrm{VO}_{2} \mathrm{max}$, fasting plasma glucose and plasma concentrations of inflammatory markers as determinants for changes in insulin sensitivity. Glucose infusion rate during the steady-state of the euglycemic-hyperinsulinemic clamp was chosen as the dependent variable in the stepwise multivariate linear regression analysis (Table 3 ). Changes in adiponectin plasma concentrations, fasting plasma glucose and percentage of body fat emerged as significant determinants of changes in insulin sensitivity after 4 weeks of physical exercise (Table 3). The following parameters did not reach statistical significance $(P>0.25)$ when included one-by-one into the model: $\Delta \mathrm{CRP}(P=0.35), \Delta \mathrm{IL}-10$ $(P=0.4), \Delta \mathrm{IL}-6(P=0.42)$ and $\Delta \mathrm{VO}_{2} \max (P=0.77)$.

\section{Discussion}

Epidemiological studies have demonstrated that physical activity is associated with reduced risk of CVD, especially in individuals with T2D $(18,24)$. The reduced CVD risk associated with exercise in patients

Table 2 Multivariate linear regression analysis of relative changes in percent body fat, $\mathrm{VO}_{2} \mathrm{max}$, and fasting plasma glucose as predictors for the of relative changes in adiponectin, IL-6, IL-10 and CRP plasma concentrations.

\begin{tabular}{lccc}
\hline & $\begin{array}{c}\Delta \% \text { Body fat } \boldsymbol{\beta} \\
(P \text {-value })\end{array}$ & $\begin{array}{c}\Delta \mathbf{V O}_{2} \max \boldsymbol{\beta} \\
(P \text {-value })\end{array}$ & $\begin{array}{c}\Delta \text { FPG } \boldsymbol{\beta} \\
(P \text {-value })\end{array}$ \\
\hline$\Delta$ Adiponectin & $-0.04(0.78)$ & $0.1(0.42)$ & $-0.26(0.04)$ \\
$\Delta$ IL-6 & $-0.09(0.5)$ & $0.06(0.7)$ & $0.09(0.5)$ \\
$\Delta$ IL-10 & $-0.08(0.53)$ & $0.21(0.1)$ & $0.26(0.04)$ \\
$\Delta$ CRP & $0.08(0.5)$ & $-0.07(0.63)$ & $0.04(0.7)$ \\
\hline
\end{tabular}

with T2D could be mediated by improved markers of inflammation and endothelial dysfunction. We therefore determined the effect of an intensive 4 week physical training on plasma concentrations of the inflammatory parameters IL-6, adiponectin, CRP and IL-10 in 60 individuals with NGT, IGT or T2D. In all three glucose-tolerance groups, fitness level, as measured by $\mathrm{VO}_{2}$ max, significantly increased after the 4 week training period. This training effect was associated with significant decreases in BMI, WHR, and percent body fat. In patients with IGT and T2D, but not in the NGT group, there was an additional effect of training on improved insulin sensitivity. The absence of improved insulin sensitivity in the NGT group was expected, because of the already high degree of insulin sensitivity in these healthy individuals.

The key finding of this study was that 4 weeks of supervised intensive regular exercise were associated with significantly improved adiponectin and CRP plasma concentrations in subjects with IGT and T2D. However, no effect of 4 weeks of exercise training on

Table 3 Stepwise multivariate regression analysis of changes in anthropometric and biochemical parameters as predictors of relative changes in glucose infusion rate (GIR) during the steadystate of euglycemic-hyperinsulinemic clamps. The following parameters did not reach statistical significance $(P>0.25)$ when included one-by-one into the model: $\Delta \mathrm{CRP}, \Delta \mathrm{IL}-10, \Delta \mathrm{IL}-6$, $\Delta \mathrm{VO}_{2} \max$.

\begin{tabular}{lccr}
\hline & \multicolumn{3}{c}{$\Delta$ GIR $(\boldsymbol{\mu m o l} / \mathbf{k g} / \mathbf{m i n})$} \\
\cline { 2 - 4 } & Step & $\beta$ & $P$-value \\
\hline$\Delta$ Adiponectin $(\mu \mathrm{g} / \mathrm{ml})$ & 1 & 0.45 & 0.004 \\
$\Delta$ FPG $(\mathrm{mmol} / \mathrm{l})$ & 2 & 0.36 & 0.026 \\
$\Delta \mathrm{BF}(\%)$ & 3 & -0.23 & 0.034 \\
\hline
\end{tabular}

$\mathrm{FPG}$, fasting plasma glucose, $\mathrm{BF}$, body fat. 
these markers of inflammation was observed in healthy individuals with NGT. This result suggests that physical training can normalize alterations in these plasma parameters associated with abnormal glucose tolerance and could therefore be due to changes in glucose or FFA levels or other metabolic abnormalities.

The increase in adiponectin plasma concentration we observed in our study after a 4 week training program is in contrast to recent studies, in which no alterations of adiponectin levels were found $(15,16)$. However, distinct characteristics of study populations and training intervention programs could explain these different results.

Our finding that exercise reduces CRP concentrations is supported by at least two longitudinal studies showing that regular training induces a reduction in CRP level $(25,26)$. However, in contrast to our results it was recently shown that in nondiabetic, insulin-resistant individuals exercise training is not associated with improved CRP or adiponectin levels (20). Moreover, Nicklas et al. (17) recently showed that exercise training did not have a significant effect on CRP and IL-6 plasma concentrations, whereas diet-induced weight loss significantly improved these parameters of chronic inflammation. Differences in the duration and intensity of training and more likely in the study population, i.e. patients with IGT or T2D in our cohort, nondiabetic subjects in one previous report (20), and older, overweight or obese patients with osteoarthrosis in the other study (17), could explain these discrepant results. The improvement in CRP (Fig. 4) and adiponectin (Fig. 2) plasma concentrations was disproportionally higher than expected from the improvement in percent body fat, $\mathrm{VO}_{2} \max$ and insulin sensitivity after training, suggesting additional accompanying effects of exercise to improve plasma concentrations of these parameters. Interestingly, the changes in CRP are not related to changes in IL-6 plasma concentrations, although CRP levels are driven to a large extent by IL-6 (27). This suggests that other factors which significantly changed with the physical training are stronger determinants of CRP plasma concentration in our intervention study.

A marked increase in circulating levels of IL-6 after exercise without muscle damage has been a remarkably consistent finding (28). Plasma IL-6 concentration increases in an exponential fashion with exercise and is related to exercise intensity, duration, the mass of muscle recruited, and endurance capacity (28). However, whether these acute effects of exercise on IL-6 concentrations lead to chronically changed IL-6 levels is unclear. In the resting state, IL-6 plasma concentrations were not significantly different between elite rowers and non-athletic controls (29), suggesting that body fitness is no major determinant of resting IL-6 plasma concentration. This is in accord with our results, that 4 weeks of exercise did not have any effect on IL-6 plasma concentrations. Despite a significant correlation of IL- 6 plasma concentration with measures of obesity, fitness level, insulin sensitivity and glucose metabolism, changes in these parameters did not predict changes in IL-6 levels (Fig. 3).

There was a trend for elevated IL-10 plasma concentrations after 4 weeks of exercise in the IGT and T2D groups, suggesting that IL-10 mediates some of the beneficial chronic effects of exercise. The increase of IL-10 levels after 4 weeks of exercise was proportional to the reduction of total body fat, the increase in fitness level and insulin sensitivity, suggesting that changes in IL-10 can be primarily explained by changes of these parameters. Moreover, multivariate regression analysis identified a decreased fasting plasma glucose concentration as a significant determinant of elevated IL-10 plasma concentration.

Subclinical inflammation is associated with insulin resistance and could thereby precede the development of T2D $(30,31)$. Interestingly, stepwise multivariate regression analysis revealed changes in adiponectin plasma concentration as the strongest predictor of changes in insulin sensitivity as measured by glucose infusion rate during the steady-state of a euglycemic-hyperinsulinemic clamp. This result further supports the concept of adiponectin as an adipokine with insulin-sensitizing effects $(32,33)$. In addition to plasma adiponectin concentrations, stepwise multivariate regression analysis identified changes in fasting plasma glucose and percent body fat as significant predictors of relative changes in glucose infusion rate during the steady-state of the euglycemic-hyperinsulinemic clamp, thereby confirming the known relationships between insulin sensitivity, hyperglycemia and adiposity. However, we could not confirm our main hypothesis that exercise-induced changes in inflammatory markers could contribute to the improvement of insulin sensitivity after physical training. In our study, changes in inflammatory parameters did not appear to be related to the improvement of insulin sensitivity, suggesting that chronic subclinical inflammation is not the predominant determinant of insulin sensitivity.

In conclusion, in patients with IGT and T2D, physical training led to a near normalization of adiponectin and CRP plasma concentrations. The increase in CRP and adiponectin was disproportionally higher than expected from the improvement in percent body fat, $\mathrm{VO}_{2}$ max and insulin sensitivity after training, suggesting additional beneficial effects of exercise on plasma concentrations of these parameters. Increased insulin sensitivity after training was most strongly related to changes in adiponectin plasma concentrations, in fasting plasma glucose and percent body fat, whereas changes in IL-6, IL-10 and CRP plasma concentrations did not significantly contribute to improved insulin sensitivity.

\section{Acknowledgements}

This study was supported by grants from the Deutsche Forschungsgemeinschaft (DFG), BL 580/3-1 (to M B) and the Bundesministerium für Bildung und Forschung 
(BMBF), Interdisciplinary Center for Clinical Research (IZKF) at the University of Leipzig (Project N 03) (to M B).

\section{References}

1 Colwell JA. Vascular thrombosis in type II diabetes mellitus. Diabetes $1993 \mathbf{4 2} 8-11$.

2 Haffner SM, Lehto S, Ronnemaa T, Pyorala K \& Laakso M. Mortality from coronary heart disease in subjects with type 2 diabetes and in nondiabetic subjects with and without prior myocardial infarction. New England Journal of Medicine 1998339 229-234.

3 Ross R. Atherosclerosis - an inflammatory disease. New England Journal of Medicine 1999340 115-126.

4 Festa A, D'Agostino R Jr, Howard G, Mykkanen L, Tracy RP \& Haffner SM. Chronic subclinical inflammation as part of the insulin resistance syndrome: the Insulin Resistance Atherosclerosis Study (IRAS). Circulation 2000102 42-47.

5 Fernandez-Real JM, Broch M, Vendrell J, Gutierrez C, Casamitjana R, Pugeat M, Richart C \& Ricart W. Interleukin-6 gene polymorphism and insulin sensitivity. Diabetes 200049 517-520.

6 Blüher M, Fasshauer M, Tönjes A, Kratzsch J, Schön MR \& Paschke R. Association of interleukin-6, C-reactive protein, interleukin-10 and adiponectin plasma concentrations with measures of obesity, insulin sensitivity and glucose metabolism. Experimental and Clinical Endocrinology and Diabetes 2005113 534-537.

7 McLaughlin T, Abbasi F, Lamendola C, Liang L, Reaven G, Schaaf P \& Reaven P. Differentiation between obesity and insulin resistance in the association with C-reactive protein. Circulation $20021062908-2912$.

8 Visser M, Bouter LM, McQuillan GM, Wener MH \& Harris TB. Elevated C-reactive protein levels in overweight and obese adults. Journal of the American Medical Association 1999282 2131-2135.

9 Weyer C, Funahashi T, Tanaka S, Hotta K, Matsuzawa Y, Pratley RE \& Tataranni PA. Hypoadiponectinemia in obesity and type 2 diabetes: close association with insulin resistance and hyperinsulinemia. Journal of Clinical Endocrinology and Metabolism 200186 1930-1935.

10 Arita Y, Kihara S, Ouchi N, Takahashi M, Maeda K, Miyagawa J, Hotta K, Shimomura I, Nakamura T, Miyaoka K, Kuriyama H, Nishida M, Yamashita S, Okubo K, Matsubara K, Muraguchi M, Ohmoto Y, Funahashi T \& Matsuzawa Y. Paradoxical decrease of an adipose-specific protein, adiponectin, in obesity. Biochemical and Biophysical Research Communications 1999257 79-83.

11 Engeli S, Feldpausch M, Gorzelniak K, Hartwig F, Heintze U, Janke J, Mohlig M, Pfeiffer AF, Luft FC \& Sharma AM. Association between adiponectin and mediators of inflammation in obese women. Diabetes $200352942-947$.

12 Esposito K, Pontillo A, Di Palo C, Giugliano G, Masella M, Marfella R \& Giugliano D. Effect of weight loss and lifestyle changes on vascular inflammatory markers in obese women: a randomized trial. Journal of the American Medical Association 2003289 1799-1804.

13 Esposito K, Pontillo A, Giugliano F, Di Palo C, Grella E, Nicoletti G \& Giugliano D. Association of low interleukin-10 levels with the metabolic syndrome in obese women. Journal of Clinical Endocrinology and Metabolism $2003 \mathbf{8 8}$ 1055-1058.

14 van Exel E, Gussekloo J, de Craen AJ, Frolich M, Bootsma-Van Der Wiel A \& Westendorp RG. Leiden 85 Plus Study: Low production capacity of interleukin-10 associates with the metabolic syndrome and type 2 diabetes: the Leiden 85-Plus Study. Diabetes $2002511088-1092$.

15 Ryan AS, Nicklas BJ, Berman DM \& Elahi D. Adiponectin levels do not change with moderate dietary induced weight loss and exercise in obese postmenopausal women. International Journal of Obesity Related Metabolic Disorders 200327 1066-1071.

16 Hulver MW, Zheng D, Tanner CJ, Houmard JA, Kraus WE, Slentz CA, Sinha MK, Pories WJ, MacDonald KG \& Dohm GL. Adiponectin is not altered with exercise training despite enhanced insulin action. American Journal of Physiology. Endocrinology and Metabolism 2002283 E861-E865.

17 Nicklas BJ, Ambrosius W, Messier SP, Miller GD, Penninx BWJH, Loeser RF, Palla S, Bleecker E \& Pahor M. Diet-induced weight loss, exercise, and chronic inflammation in older, obese adults: a randomized controlled clinical trial. American Journal of Clinical Nutrition 200479 544-551.

18 Tanasescu M, Leitzmann MF, Rimm EB \& Hu FB. Physical activity in relation to cardiovascular disease and total mortality among men with type 2 diabetes. Circulation $2003 \mathbf{1 0 7} 2435-2439$.

19 Kasapis C \& Thompson PD. The effects of physical activity on serum C-reactive protein and inflammatory markers: a systematic review. Journal of the American College of Cardiology $2005 \mathbf{4 5}$ $1563-1569$.

20 Marcell TJ, McAuley KA, Traustadottir T \& Reaven PD. Exercise training is not associated with improved levels of C-reactive protein or adiponectin. Metabolism 2005 54 533-541.

21 Report of the Expert Committee on the Diagnosis and Classification of Diabetes Mellitus. Diabetes Care 200023 S4-S19.

22 Blüher M, Unger R, Rassoul F, Richter V \& Paschke R. Relation between glycaemic control, hyperinsulinaemia and plasma concentrations of soluble adhesion molecules in patients with impaired glucose tolerance or Type II diabetes. Diabetologia 200245 210-216.

23 DeFronzo RA, Tobin JD \& Andres R. Glucose clamp technique: a method for quantifying insulin secretion and resistance. American Journal of Physiology 1979237 E214-E223.

24 Stewart KJ. Exercise training: can it improve cardiovascular health in patients with type 2 diabetes? British Journal of Sports Medicine 200438 250-252.

25 Fallon KE, Fallon SK \& Boston T. The acute phase response and exercise: court and field sports. British Journal of Sports Medicine $200135170-173$.

26 Mattusch F, Dufaux B, Heine O, Mertens I \& Rost R. Reduction of the plasma concentration of C-reactive protein following nine months of endurance training. International Journal of Sports Medicine $20002121-24$.

27 Yudkin JS, Kumari M, Humphries SE \& Mohamed-Ali V. Inflammation, obesity, stress and coronary heart disease: is interleukin-6 the link? Atherosclerosis $2000 \mathbf{1 4 8} 209-214$.

28 Petersen AM \& Pedersen BK. The anti-inflammatory effect of exercise. Journal of Applied Physiology 200598 1154-1162.

29 Nieman DC, Nehlsen-Cannarella SL, Fagoaga OR, Henson DA, Shannon M, Hjertman JM, Schmitt RL, Bolton MR, Austin MD, Schilling BK \& Thorpe R. Immune function in female elite rowers and non-athletes. British Journal of Sports Medicine 2000 $34181-187$.

30 Schmidt MI, Duncan BB, Sharrett AR, Lindberg G, Savage PJ, Offenbacher S, Azambuja MI, Tracy RP \& Heiss G. Markers of inflammation and prediction of diabetes mellitus in adults (Atherosclerosis Risk in Communities study): a cohort study. Lancet $19993531649-1652$

31 Pradhan AD, Manson JE, Rifai N, Buring JE \& Ridker PM. C-reactive protein, interleukin 6 , and risk of developing type 2 diabetes mellitus. Journal of the American Medical Association 2001286 327-334.

32 Berg AH, Combs TP, Du X, Brownlee M \& Scherer PE. The adipocyte-secreted protein Acrp30 enhances hepatic insulin action. Nature Medicine $20017947-953$.

33 Yamauchi T, Kamon J, Waki H, Terauchi Y, Kubota N, Hara K, Mori Y, Ide T, Murakami K, Tsuboyama-Kasaoka N, Ezaki O, Akanuma Y, Gavrilova O, Vinson C, Reitman ML, Kagechika H, Shudo K, Yoda M, Nakano Y, Tobe K, Nagai R, Kimura S, Tomita M, Froguel P \& Kadowaki T. The fat-derived hormone adiponectin reverses insulin resistance associated with both lipoatrophy and obesity. Nature Medicine $20017941-946$.

Received 18 November 2005

Accepted 19 January 2006 